\title{
Targeting Pericytes Diminishes Neovascularization in Orthotopic Uveal Melanoma in Nerve/Glial Antigen 2 Proteoglycan Knockout Mouse
}

\section{Ugur Ozerdem}

La Jolla Institute for Molecular Medicine, San Diego, Calif., USA

\section{Key Words}

Angiogenesis • Uveal melanoma • Microcirculation •

Neovascularization $\cdot$ Pericyte pericyte component, inhibited neovascularization and growth of uveal melanoma xenografts. For this, we used multichannel laser scanning confocal microscopy and quantitative image analysis.

\section{Methods}

NG2 null mice were generated via a conventional homologous recombination approach. The mice were back-crossed onto a C57BL/6 genetic background, and NG2+/- heterozygotes were mated to establish separate NG2 knockout (NG2-/-) and wildtype $(\mathrm{NG} 2+/+)$ colonies.

The OCM-1A human uveal melanoma cell line was used to induce orthotopic uveal melanoma xenografts. Beginning 3 days prior to surgery, $60 \mathrm{mg} / \mathrm{kg}$ cyclosporin A was administered daily via intraperitoneal injections. We used a similar microsurgical technique described previously for inducing uveal melanoma xenografts [1] with several modifications: a $5-\mu$ l suspension of $5 \times$ $10^{5}$ OCM-1A cells was injected into the suprachoroidal space $1.5 \mathrm{~mm}$ posterior to the corneoscleral limbus. Arrows in figure 1a indicate the route (tunnel) of microinjection. The mice were enucleated 2 weeks after surgery.

Immunohistochemistry, Confocal Microscopic Imaging, and Image Analysis

Blood vessel endothelial cells were identified using a cocktail of rat antibodies against mouse endoglin (CD105), PECAM-1 (CD31), and VEGF receptor-2 (flk-1; Pharmingen, San Diego, Calif., USA). Pericytes were identified by labeling with rabbit PDGF $\beta$-receptor antibodies, and rabbit or guinea pig anti-

\section{KARGER}

() 2006 S. Karger AG, Basel

Fax +41613061234 E-Mail karger@karger.ch www.karger.com www.karger.com/ore
Ugur Ozerdem, MD, Assistant Professor La Jolla Institute for Molecular Medicine

4570 Executive Drive, Suite 100, San Diego, CA 92121 (USA)

Tel. +1 858587 8788, ext. 128, Fax +1 8585876742

E-Mail ozerdem@ljimm.org 

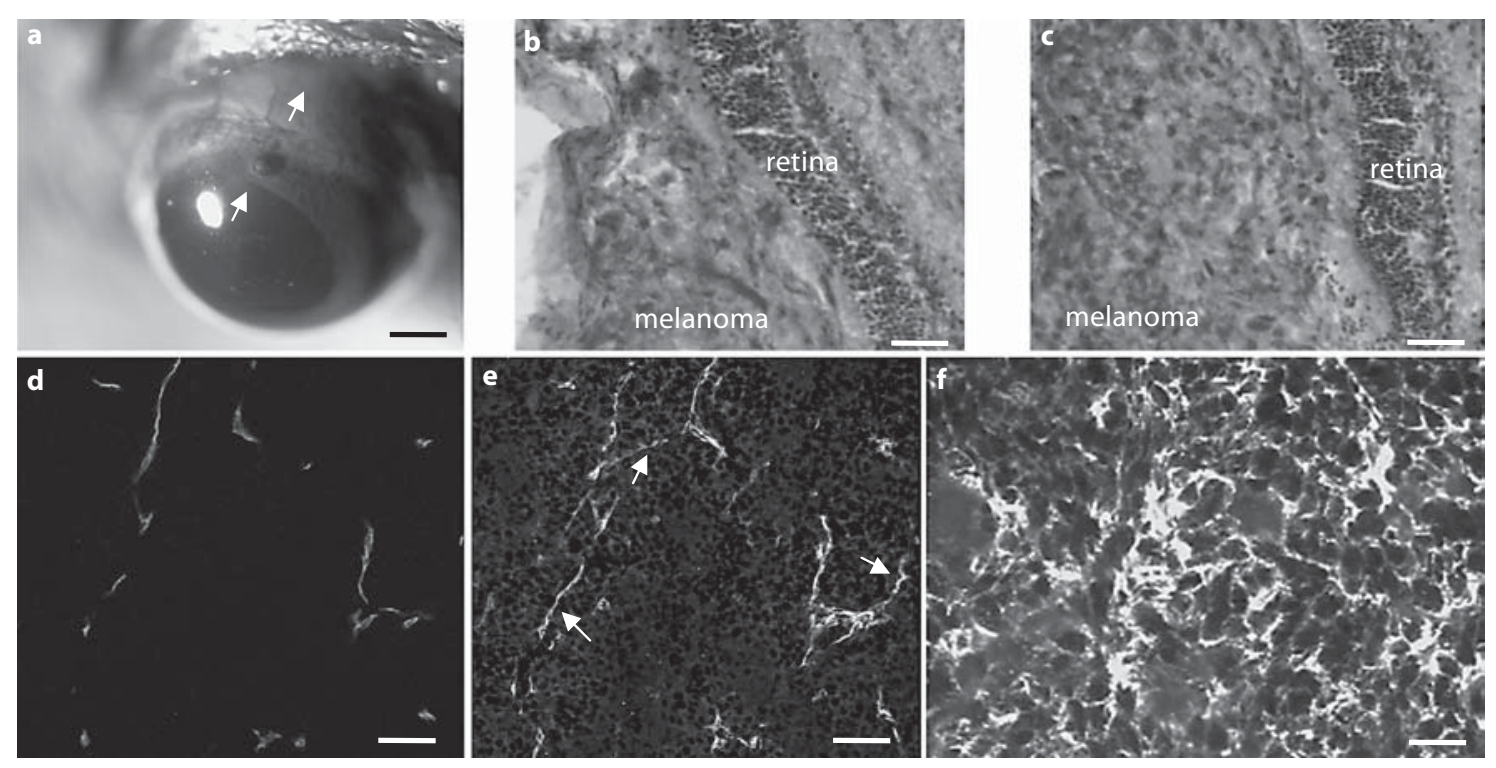

Fig. 1. OCM-1A cells were inoculated into suprachoroidal spaces of cyclosporin A-immunosuppressed mice. Arrows indicate the direction of the tangential tunnel prepared through the corneoscleral junction and the sclera. a Two weeks following surgery, anterior segments, lens, and vitreous look clear, with a patchy area of iris atrophy near the limbus. Both NG2 knockout (b) and wildtype (c) eyes developed tumors in 2 weeks in the choroid between the sclera and retina. Frozen sections of OCM-1A tumor grafts grown in NG2 knockout and wild-type mice were double-immunostained and imaged with a scanning laser confocal microscope. Blood vessel endothelium was identified using a cocktail of antibodies against endoglin (CD105), PECAM-1 (CD31) and VEGF receptor-2 (flk-1) (d), and pericytes were identified using PDGF $\beta$-receptor antibodies (e). d, e Histological sections from a wildtype mouse and double immunostained for endothelium markers $(\mathrm{CD} 105+\mathrm{CD} 31+\mathrm{flk}-1)(\mathbf{d})$, and a pericyte marker PDGF $\beta$-receptor (e). Confocal microscopy revealed extensive investment of endothelium with pericytes. e Pericytes were easily identified in neovascular walls, and were also found to be forming segments of vessels not lined with endothelium (arrows). f OCM-1A tumor cells with NG2 expression in an NG2 knockout mouse. OCM-1A tumor cells showed NG2 expression. Scale bars indicate $750 \mu \mathrm{m}$ (a), $100 \mu \mathrm{m}(\mathbf{b}, \mathbf{c}), 75 \mu \mathrm{m}(\mathbf{d}, \mathbf{e}), 30 \mu \mathrm{m}(\mathbf{f})$. bodies against the NG2 proteoglycan [2]. Rabbit polyclonal glial fibrillary acidic protein (Accurate Chemical, Westbury, N.Y., USA) and PDGF $\alpha$-receptor antibodies (a kind gift from Dr. William B. Stallcup) were used to identify glia. Slides incubated with nonspecific immunoglobulin served as a negative control.

The immunostained sections ( 3 sections per eye from 18 eyes) were analyzed with an Olympus Fluoview 1000 multi-channel laser scanning confocal microscope for microvascular density (MVD). The Volocity image analysis software (Openlab-Improvision Inc, Lexington, Mass., USA) was used for quantification of MVD in pixels in sections immunostained with endoglin (CD105), PECAM-1 (CD31) and VEGF receptor-2 (flk-1). The average equatorial radius $(\mathrm{R})$ of each tumor in hematoxylin and eosinstained histological slides was measured by using the Volocity image analysis software. Then the volume of each tumor was estimated using the formula $4 / 3 \times \pi \times R^{3}$.

\section{Results}

On dilated indirect ophthalmoscopic examination, the tumor mass was identified at the peripheral fundus. The visual axis was intact, and the anterior chamber, cornea, lens, and vitreous were clear (fig. 1a). Both NG2 knockout (fig. 1b) and wild-type (fig. 1c) eyes developed tumors in uvea.

Immunostaining for NG2 and PDGF $\beta$-receptors revealed NG2-negative and PDGF $\beta$-receptor-positive pericytes in NG2 knockout mice and NG2-positive and PDGF $\beta$-receptor-positive pericytes in the wild-type mice. PDGF $\beta$-receptor and [endoglin (CD105), PECAM-1 (CD31), and VEGF receptor-2 (flk-1)] double immunostaining revealed investment of endothelium with pericytes (fig. 1d, e). OCM-1A tumor cells showed NG2 expression, both in NG2 knockout (fig. 1f) and wild-type mice. The mean vascular density was 153.3 

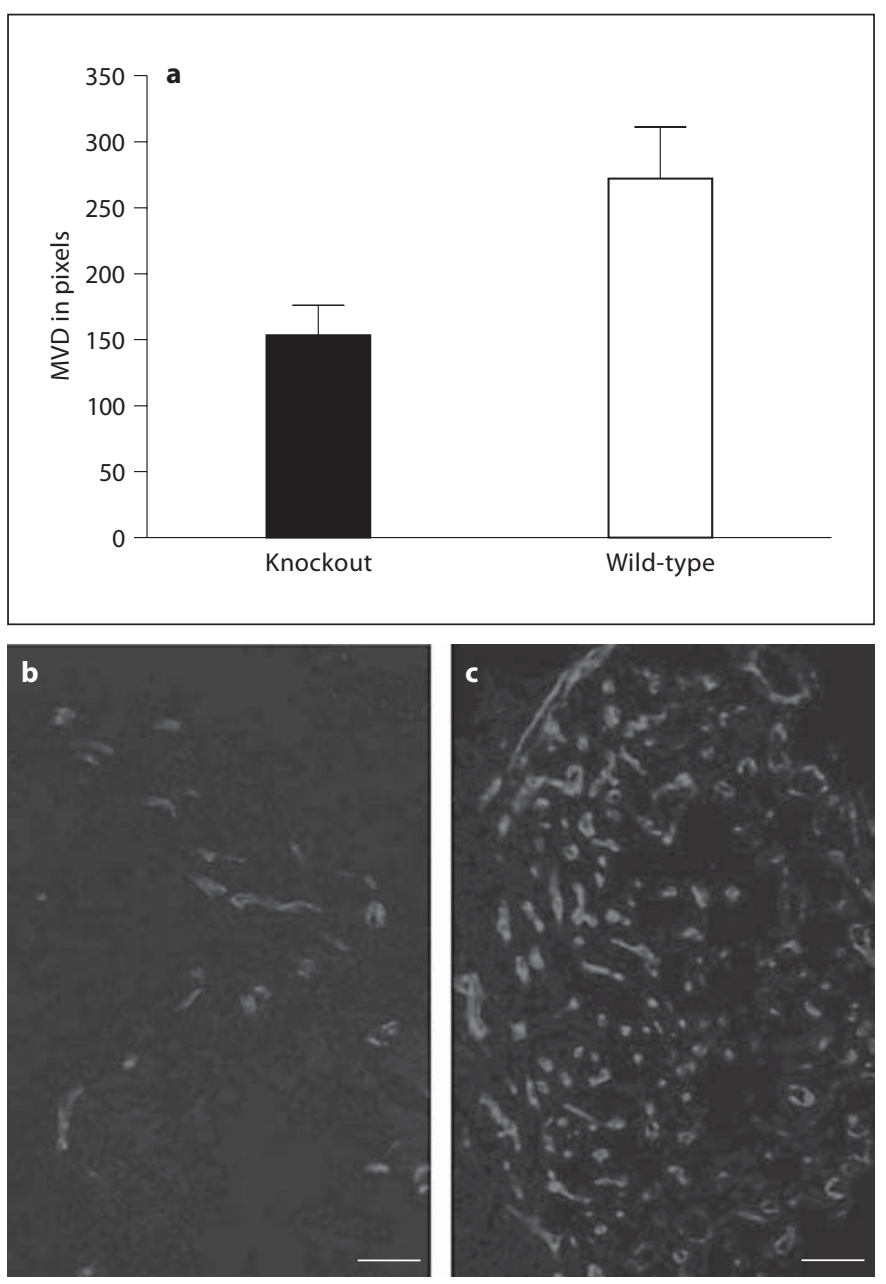

Fig. 2. MVD was quantified in pixels using Volocity image analysis software on 54 frozen sections which were sampled from 18 eyes, and immunostained for endoglin (CD105), PECAM-1 (CD31), and VEGF receptor-2 (flk-1). a MVD in NG2 knockout eyes was $43.7 \%$ lower than in wild-type controls, and this difference was statistically significant ( $\mathrm{n}=18$ eyes, $\mathrm{p}=0.0166$, MannWhitney U test). b MVD in uveal melanoma in a knockout eye. Scale bar indicates $75 \mu \mathrm{m}$. c Tumor in a control eye (wild-type). Scale bar indicates $75 \mu \mathrm{m}$.

pixels in NG2 knockout eyes (standard error of mean \pm 22.7 ) and 272.3 pixels in wild-type eyes (standard error of mean \pm 38.7). The mean MVD in NG2 knockout eyes was therefore $43.7 \%$ lower than in wild-type controls, and this difference was statistically different ( $\mathrm{n}=$ 18 eyes, $p=0.0166$, Mann-Whitney $U$ test; fig. $2 \mathrm{a}$ ). We have not encountered any cells within uveal melanoma showing glial or oligodendroglial differentiation by using glial fibrillary acidic protein or PDGF- $\alpha$ receptor immunohistochemistry. The mean tumor end volume in the knockout and wild-type mice was $0.1873 \mathrm{~mm}^{3}$ and $3.6262 \mathrm{~mm}^{3}$, respectively $(\mathrm{n}=18, \mathrm{p}<0.0001 \mathrm{Mann}$ Whitney U test).

\section{Discussion}

The use of the xenograft model and cyclosporin administration for immunosuppression may somewhat confound the interpretation of the results in the context of tumor-host interactions and neovascular cell recruitment. The cyclosporin inhibits angiogenesis by inhibiting migration of vascular precursors. This effect is mediated through the inhibition of cyclooxygenase 2 , the transcription of which is modulated by the nuclear factor of activated $\mathrm{T}$ cells [3]. Therefore, immunosuppression of the mice in order to prevent rejection of the human tumor xenograft, although necessary, presents a significant problem. However, four observations presented in our study seem especially noteworthy.

First, pericytes contribute to the neovascular vessels of uveal melanoma extensively. To our knowledge, there are at least two reports on the presence of $\alpha$-smooth muscle actin-positive pericytes in uveal melanoma $[4,5]$. Since only a small fraction (1-10\%) of nascent pericytes can be identified on the basis of ASMA expression [6], we used NG2 and PDGF $\beta$-receptor immunohistochemistry to identify pericytes.

The current investigation represents the first report on therapeutic targeting of pericytes in uveal melanoma. Presence of NG2-negative and PDGF $\beta$-receptor-positive pericytes in uveal melanoma in NG2 knockout mice also suggests that neovascular pericytes in uveal melanoma derive from the host tissue, but not from the grafted tumor.

Second, genetic inhibition of pericyte-NG2 proteoglycan decreases MVD in uveal melanoma. NG2, a membrane-spanning chondroitin sulfate proteoglycan associated with mitotically active, nascent pericytes exhibits several properties, which suggests that it is a functional player in neovascularization [7, 8]. NG2 appears to serve as a coreceptor for bFGF (basic fibroblast growth factor) [9]. Recent investigations have revealed decreased neovascularization following intrinsic or extrinsic targeting of NG2 proteoglycan in retinal ischemia, neurofibromatosis type 1 , in bFGF-induced neovascularization in cornea.

Third, the reduction of the MVD leads to a significant inhibition of uveal melanoma growth. These results sug- 
gest a $95 \%$ reduction of the tumor end volume at the time of sacrifice in NG2 knockout mice.

Fourth, expression of NG2 proteoglycan on both pericytes and uveal melanoma cells renders this proteoglycan a double target.

The current study expands our understanding of the neovascularization process in uveal melanoma xenografts and establishes a role for pericytes and NG2 proteoglycan as potential therapeutic targets.

\section{Acknowledgments}

This work has been supported by NIH (RO3 HD044783), University of California (TRDRP 13IT-0067), and the DoD-CDMRP (PC020822).

\section{References}

1 Hu LK, Huh K, Gragoudas ES, Young LH: Establishment of pigmented choroidal melanomas in a rabbit model. Retina 1994;14: 264-269.

2 Ozerdem U, Alitalo K, Salven P, Li A: Contribution of bone marrow-derived pericyte precursor cells to corneal vasculogenesis. Invest Ophthalmol Vis Sci 2005;46:3502-3506.

>3 Hernandez GL, Volpert OV, Iniguez MA, Lorenzo E, Martinez-Martinez S, Grau R, Fresno M, Redondo JM: Selective inhibition of vascular endothelial growth factor-mediated angiogenesis by cyclosporin A: roles of the nuclear factor of activated T cells and cyclooxygenase 2. J Exp Med 2001;193:607620 .
4 Makitie T, Summanen P, Tarkkanen A, Kivela $\mathrm{T}$ : Microvascular density in predicting survival of patients with choroidal and ciliary body melanoma. Invest Ophthalmol Vis Sci 1999;40:2471-2480.

5 Clarijs R, van Dijk M, Ruiter DJ, de Waal RM: Functional and morphologic analysis of the fluid-conducting meshwork in xenografted cutaneous and primary uveal melanoma. Invest Ophthalmol Vis Sci 2005;46: 3013-3020.

6 Balabanov R, Dore-Duffy P: Role of the CNS microvascular pericyte in the blood-brain barrier. J Neurosci Res 1998;53:637-644.
Ozerdem U: Targeting neovascular pericytes in neurofibromatosis type 1 . Angiogenesis 2004;7:307-311.

8 Ozerdem U, Stallcup WB: Pathological angiogenesis is reduced by targeting pericytes via the NG2 proteoglycan. Angiogenesis 2004;7:269-276.

9 Goretzki L, Burg MA, Grako KA, Stallcup WB: High-affinity binding of basic fibroblast growth factor and platelet- derived growth factor-AA to the core protein of the NG2 proteoglycan. J Biol Chem 1999;274: 16831-16837. 